\title{
BMJ Open Safety, feasibility and efficacy of metformin and sitagliptin in patients with a TIA or minor ischaemic stroke and impaired glucose tolerance
}

\author{
Elizabeth Osei (D) , ${ }^{1}$ Adrienne Zandbergen, ${ }^{2}$ Paul J A M Brouwers, ${ }^{3}$ \\ Laus J M M Mulder, ${ }^{4}$ Peter Koudstaal, ${ }^{5}$ Hester Lingsma, ${ }^{6}$ Diederik W J Dippel (D) , \\ Heleen den Hertog ${ }^{7}$
}

To cite: Osei E, Zandbergen A, Brouwers PJAM, et al. Safety, feasibility and efficacy of metformin and sitagliptin in patients with a TIA or minor ischaemic stroke and impaired glucose tolerance. BMJ Open 2021;11:e046113. doi:10.1136/ bmjopen-2020-046113

- Prepublication history for this paper is available online. To view these files, please visit the journal online (http://dx.doi org/10.1136/bmjopen-2020046113).

Received 22 0ctober 2020 Accepted 26 July 2021

Check for updates

(c) Author(s) (or their employer(s)) 2021. Re-use permitted under CC BY-NC. No commercial re-use. See rights and permissions. Published by BMJ.

${ }^{1}$ Neurology, Flevoziekenhuis, Almere, The Netherlands ${ }^{2}$ Internal Medicine, Erasmus MC, Rotterdam, The Netherlands ${ }^{3}$ Neurology, Medisch Spectrum Twente, Enschede, The Netherlands

${ }^{4}$ Neurology, Ikazia Hospital, Rotterdam, The Netherlands ${ }^{5}$ Neurology, Erasmus MC, Rotterdam, The Netherlands

${ }^{6}$ Public Health, Erasmus MC, Rotterdam, The Netherlands ${ }^{7}$ Neurology, Isala Hospitals, Zwolle, The Netherlands

Correspondence to

Dr Elizabeth Osei;

I.osei.neurologie@gmail.com

\section{ABSTRACT}

Introduction Impaired glucose tolerance (IGT) is highly prevalent after stroke and is associated with recurrent stroke and unfavourable outcome.

Objectives We aimed to assess the feasibility, safety and effects on glucose metabolism of metformin or sitagliptin in patients with transient ischaemic attack (TIA) or minor ischaemic stroke and IGT.

Design We performed a multicentre, randomised, controlled, open-label phase II trial with blinded outcome assessment.

Interventions Patients were randomised in a 2:1:1 ratio to 'no medication', sitagliptin or metformin.

Primary and secondary outcome measures Primary outcome measures were baseline adjusted differences of 2-hour postload glucose; secondary outcome measures fasting glucose, glycosylated haemoglobin 1c (HbA1C) levels, tolerability and safety of metformin and sitagliptin at 6 months. Patients on metformin or sitagliptin were contacted by telephone for recording of possible adverse events and to support continuation of treatment at 2 weeks, 6 weeks and 3 months after inclusion. These events were not analysed as outcome measures.

Results Fifty-three patients were randomised to control group, 26 to metformin and 22 to sitagliptin. We found no significant differences in 2-hour postload glucose between patients on antidiabetic drugs and controls $((-0.04 \mathrm{mmol} / \mathrm{L}$ $(95 \% \mathrm{Cl}-0.53$ to 0.45$))$. Patients in the treatment arms had reduced fasting glucose: $((-0.21 \mathrm{mmo} / \mathrm{L}(95 \% \mathrm{Cl}$ -0.36 to -0.06$))$ and $\mathrm{HbA} 1 \mathrm{c}$ levels $((-1.16 \mathrm{mmol} / \mathrm{mol}$ $(95 \% \mathrm{Cl}-1.84$ to -0.49$))$. Thirteen patients (50\%) on metformin and $7(32 \%)$ on sitagliptin experienced side effects. Sixteen patients (61\%) in the metformin and 13 $(59 \%)$ in the sitagliptin group were still on treatment after 6 months.

Conclusions Metformin and sitagliptin were both effective in reducing fasting glucose and $\mathrm{HbA1c}$ levels in patients with recent TIA or minor ischaemic stroke and IGT. However, the reduction of glucose levels and sample size was relatively small. The clinical relevance, therefore, needs to be tempered. A phase III trial is needed to investigate whether medical treatment, compared with lifestyle intervention or a combination of both, not only improves glucose metabolism in IGT, but also leads to reduction of recurrent TIA or ischaemic stroke in these patients.
Strengths and limitations of this study

- Our study was a randomised, open-label controlled trial with blinded outcome assessment.

- A relatively large proportion of patients discontinued medication despite support with frequent telephone calls.

- We did not study the effectiveness of the newer GLP1 analogues or SGLT2 inhibitors, which appeared to be more promising drugs to improve glucose metabolism as well as cardiovascular outcome.

- The oral glucose tolerance test was repeated after 2-12 weeks to exclude the acute phase effect.

- Multiple glucose tests were used to determine disturbed glucose tolerance.

Trial registration number NL3048.

\section{INTRODUCTION}

Impaired glucose tolerance (IGT) refers to a metabolic state that precedes diabetes mellitus, and is highly prevalent in patients with transient ischaemic attack (TIA) or ischaemic stroke. ${ }^{1-4}$ IGT can be transient, indicating an acute stress response or persistent, reflecting undiagnosed impaired glucose metabolism. ${ }^{1}$ In non-diabetic stroke patients with IGT in the acute phase after stroke and repeated glucose assessment after 3 months, $22 \%-47 \%$ had persistent IGT. ${ }^{15-7}$ IGT is associated with recurrent stroke, other cardiovascular events, poor functional outcome and mortality in stroke patients. ${ }^{8-11}$ The mechanisms underlying this association are not fully understood, but may include endothelial dysfunction, chronic inflammation, hypercoagulability and impaired fibrinolysis. ${ }^{12-14}$

IGT is often part of the metabolic syndrome, and is associated with the individual components of this cluster of clinical abnormalities: central obesity, hypertension, 
hypertriglyceridaemia and low HDL. Therefore, IGT can be considered to represent a disturbed metabolism to a greater extend. ${ }^{15}$

Tight glycaemic control might reduce the risk of stroke in patients with diabetes or IGT. A recent randomised controlled trial showed that pioglitazone can prevent stroke and myocardial infarction among patients with insulin resistance after ischaemic stroke or TIA, but pioglitazone also gave a higher risk of weight gain, oedema and fractures. ${ }^{16} \mathrm{~A}$ meta-analysis of glucose-lowering pharmacological interventions in patients with IGT found no favourable effects on all-cause mortality or death due to major cardiovascular events, with the possible exception of stroke. ${ }^{17}$

Metformin is recommended as first-line treatment in type 2 diabetes mellitus; it is one of the most effective oral drugs in the management of type 2 diabetes, and is cheap compared with the newer antidiabetic drugs. Results from our recent study suggest that metformin treatment is safe in patients with TIA or ischaemic stroke and IGT, and probably leads to improved glucose tolerance. ${ }^{18}$ However, $50 \%$ of the patients experienced gastrointestinal side effects leading to permanent discontinuation in $25 \%$. More gradual increase in dose of metformin and better information and support on the temporary nature of the side effects might diminish the high incidence of side effects and discontinuation of treatment.

At the start of our study, selective dipeptidyl peptidase-4 (DPP-4) inhibitors were considered to have fewer side effects than metformin. Sitagliptin, a selective DPP-4 inhibitor, improves glycaemic control and $\beta$-cell function and has a safety profile similar to placebo, with low risk of gastrointestinal side effects. Also, it is associated with weight loss and a lower risk of hypoglycaemic. ${ }^{19} 20$ Currently, glucagon-like peptide 1 (GLP1) analogues or sodium-glucose co-transporter-2 (SGLT2) inhibitors are the promising newer antidiabetic medication, which have a glucose lowering effect and also can lower cardiovascular risk. ${ }^{21}{ }^{22}$ However, these medications were not readily available at the start of our study.

However, several trials showed that lifestyle modification is at least as effective as drug treatment, ${ }^{23}{ }^{24}$ but more difficult to sustain.

The aim of the study was to explore the feasibility, safety and effects on glucose metabolism of both metformin and sitagliptin in patients with TIA or minor ischaemic stroke and IGT. Considering our previous study, ${ }^{18}$ we also assessed whether a more gradual increase in dose of metformin and better support and information on this treatment reduce the incidence of side effects in these patients.

\section{MATERIALS AND METHODS}

\section{Study design}

The design of the metformin and sitagliptin in patients with impAired glucose tolerance and a recent TIA or minor ischaemic Stroke (MAAS) trial has been reported earlier. ${ }^{25}$ In summary, the MAAS-trial is a phase II, prospective, randomised, open-label, blinded end point (PROBE) and multicentre trial of standard care plus metformin or sitagliptin, as compared with standard care without anti-diabetic treatment. The study was conducted in two stroke centres in the Netherlands from 2014 to 2019.

\section{Study population}

Patients were eligible for inclusion if they were 18 years or older and had a clinical diagnosis of TIA (defined as a transient episode of neurological dysfunction caused by focal brain, spinal cord or retinal ischaemia, without acute infarction on CT scan or MRI scan), amaurosis fugax (transient monocular visual loss associated with thromboembolic events, which was also categorised as TIA) or minor ischaemic stroke (defined as brain or retinal cell death attributable to ischaemia, based on imaging or clinical objective evidence of cerebral or retinal focal ischaemic injury in a defined vascular distribution, and other etiologies excluded, with a modified Rankin scale (mRS) score of 3 or less ${ }^{26}$ within the previous 6 months. The mRS is a scale for measuring the degree of disability or dependence in the daily activities of patients. The scale runs from 0 (no symptoms) to 6 (death). $\mathrm{mRS}$ score of 3 describes patients with moderate disability, who require some help, but can walk unassisted. The diagnosis TIA (symptoms <24hours) or ischaemic stroke was made by a neurologist according to standard guidelines. All patients underwent a CT scan of the brain to confirm the diagnosis.

Eligible patients were required to have IGT, defined as 2-hour postload glucose levels between 7.8 and $11.0 \mathrm{mmol} / \mathrm{L}$ after standard oral glucose tolerance test (OGTT), which was performed on the day of the visit to the TIA outpatient clinic or on the first day after admission on the stroke unit. ${ }^{27}{ }^{28}$ The glucose levels were measured by application of a drop of blood from a finger prick to a disposable test strip, which was then inserted into an electronic blood glucose meter. The fasting glucose levels were also assessed in the laboratory. We previously stated in the study protocol that the OGTT should be repeated after 2-6 weeks to rule out laboratory error and the acute phase effect. Due to logistic reasons, this time frame was broadened to 2-12 weeks. If the second OGTT confirmed the diagnosis of IGT, and all the selection criteria were fulfilled, the patients were asked for written informed consent by the investigators. Patients were excluded if they had a history of diabetes mellitus, defined as the use of oral or parenteral antidiabetic medication. Other exclusion criteria included a history of diabetic ketoacidosis, symptoms of type 1 diabetes, signs of renal impairment, known liver disease or disturbed liver function tests, history of lactic acidosis, heart failure, pancreatitis, chronic hypoxic lung disease stage III-IV, digoxin use, pregnancy or breast feeding (for detailed list of inclusion and exclusion criteria, see study protocol) ${ }^{25}$ 


\section{Patient and public involvement}

Patients were not involved in the design, or conduct, or reporting, or dissemination plans of our research.

\section{Study procedures}

Before randomisation and at 6 months, all patients underwent OGTT with $75 \mathrm{~g}$ of glucose. At baseline, data on clinical features of TIA or ischaemic stroke, demographic data, medical history, vascular risk factors and medication use were obtained. Fasting glucose levels, glycosylated haemoglobin 1c (HbA1c) levels, body mass index (BMI), blood pressure and lipid profile were also assessed at baseline and at 6 months.

Patients were randomised to receive either open-label metformin or sitagliptin or 'no medication' in a 1:1:2 ratio for 6 months. The treatment assignment was concealed. Patients allocated to metformin started with $500 \mathrm{mg}$ two times per day, which was gradually increased in a 6-week period to $1000 \mathrm{mg}$ two times per day (week 1: 2 times $500 \mathrm{mg}$, week 3: 2 times $850 \mathrm{mg}$, week 7: 2 times $1000 \mathrm{mg}$ ). If intolerable side effects for the patients occurred after increasing the dose, the previous dose was resumed and a second challenge was performed in the next week. Patients allocated to sitagliptin were treated with a fixed daily dose of $100 \mathrm{mg}$.

Patients on metformin or sitagliptin were contacted by telephone for recording of possible adverse events and to support continuation of treatment at 2 weeks, 6 weeks and 3 months after inclusion. These events were not analysed as outcome measures. At the follow-up visit at 6 months, patients were asked to complete a single questionnaire to assess compliance and nature of any of the side effects of the study medication.

Irrespective of treatment allocation, patients received usual care from the neurologist, including antithrombotic and antihypertensive agents as well as cholesterol lowering drugs, where appropriate. ${ }^{29}$ In addition, a stroke nurse specialist provided general lifestyle advice concerning diet, smoking and physical exercise.

\section{Study outcomes}

The primary efficacy outcome was the level of 2-hour postload plasma glucose. Safety and acceptability outcomes were the number of adverse events and serious adverse events, and the number of patients still on treatment after 6 months.

Secondary outcomes were the fasting plasma glucose levels, the BMI at 6 months, presence of normal glucose tolerance at 6 months. Moreover, we studied HbA1c levels, low-density lipoprotein (LDL) levels, systolic and diastolic blood pressure at 6 months. Also, the percentage of patients with normalised fasting glucose (defined as fasting plasma glucose levels $<5.6 \mathrm{mmol} / \mathrm{L}$ ) and normalised HbAlc levels (defined as HbA1c levels $<39 \mathrm{mmol} / \mathrm{mol}$ ) at 6 months was assessed. Outcome assessment was blinded for treatment allocation.

\section{Statistical analyses}

We estimated that a sample size of 100 patients, (25 on metformin, 25 on sitagliptin and 50 in the control group) would provide a power of $80 \%$, to detect a statistically significant (alpha <0.05) effect, based on an assumed difference of $8 \%$ in 2-hour postload glucose level after 6 months between treatment and controls, assuming a mean glucose level of $9.0 \mathrm{mmol} / \mathrm{L}$ in the control group, with an $\mathrm{SD}$ of $1.0 \mathrm{mmol} / \mathrm{L}$.

The primary effect analysis estimated the difference in 2-hour postload glucose levels at 6 months between the three groups, adjusted for baseline glucose level. Analyses were done by intention to treat and all patients who were randomised to a study arm were included in the prespecified analyses.

We estimated the baseline adjusted differences of 2 hour postload glucose levels, fasting glucose levels, HbA1c levels, BMI, LDL levels and systolic and diastolic blood pressure between treatment groups with $95 \%$ CIs with multivariable linear regression, but report unadjusted analyses as well. We previously stated in our protocol that adjustments were made with a multivariable linear regression model that included the following factors: age, sex, time to treatment and baseline waist circumference. We later, but before closure of the database, decided to add baseline glucose levels to the adjustments and replace baseline waist circumference by baseline BMI. ${ }^{30}$

We compared the percentage of patients still on treatment after 6 months, the incidence of (serious) adverse events and percentage of patients with a normal glucose tolerance, normal fasting glucose and normal HbAlc levels at 6 months between treatment groups with $\chi^{2}$ test of independence. A $\mathrm{p}<0.05$ was considered to indicate statistical significance.

Furthermore, we expressed the association of the treatment groups with normalised glucose tolerance, fasting glucose and HbAlc levels with ORs and corresponding CI. These estimates were also made with multivariable logistic regression and adjusted for age, sex, time to treatment, BMI and baseline glucose levels.

In addition, subgroup analyses were performed in patients who used metformin $1000 \mathrm{mg}$ two times per day, and patients with a combination of IGT, impaired fasting glucose and impaired HbA1c levels at randomisation. We also performed an 'on-treatment analysis'. The analysis was performed using STATA V.12.1 statistical package (StataCorp).

\section{RESULTS}

A total of 263 patients with IGT based on the first OGTT underwent a repeated OGTT. Of these patients, 162 $(62 \%)$ had normalised IGT. The remaining 101 patients were included in our study. Fifty-three patients were allocated to the control group, 26 patients to metformin and 22 patients to sitagliptin.

The baseline characteristics of the enrolled patients are described in table 1 . 
Table 1 Baseline characteristics

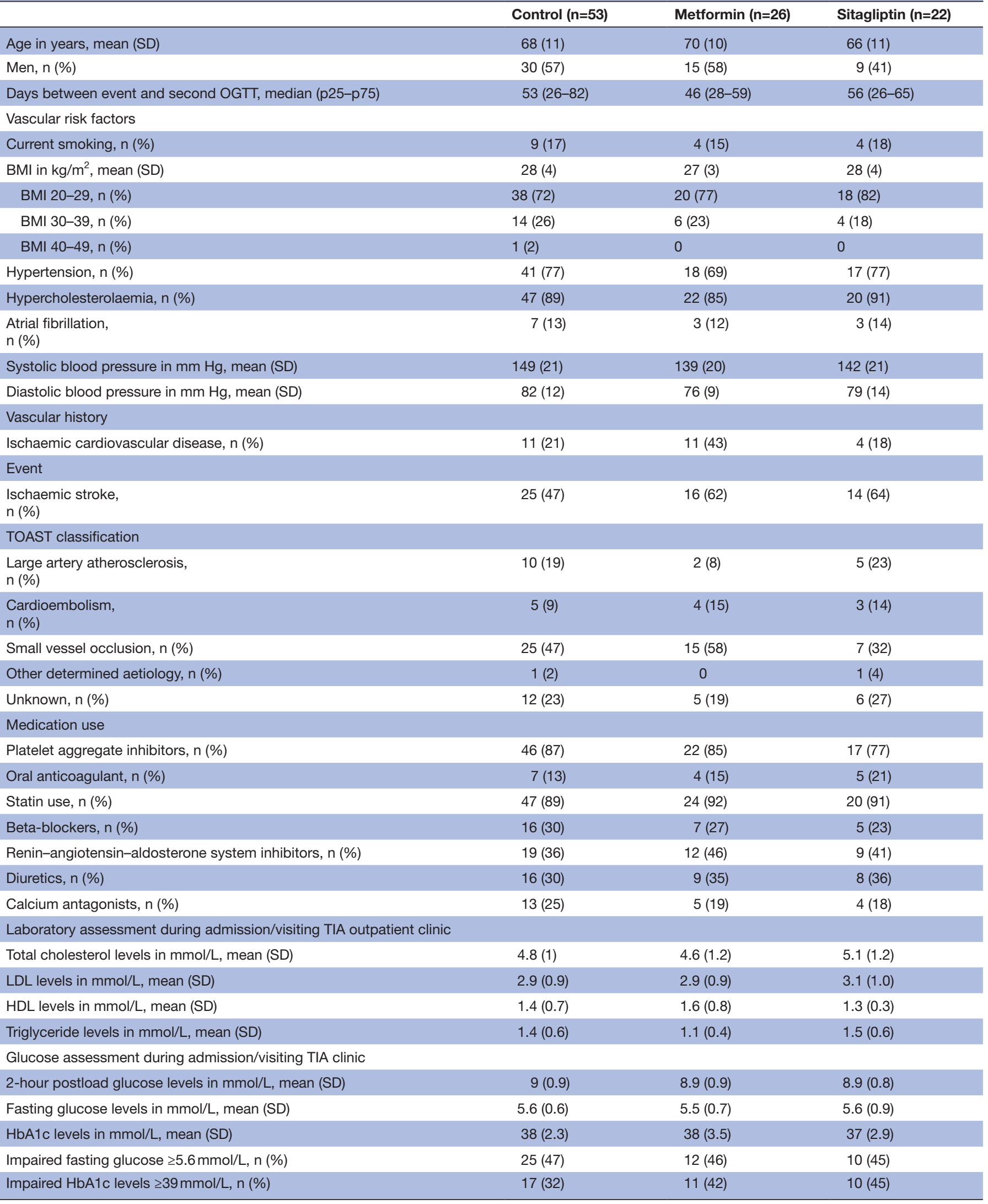

BMI, body mass index; HbA1c, glycosylated haemoglobin 1c; HDL, high-density lipoprotein; LDL, low-density lipoprotein; OGTT, oral glucose tolerance test; ; TIA, transient ischaemic attack; TOAST, Trial of Org 10172 in Acute Stroke Treatment. 


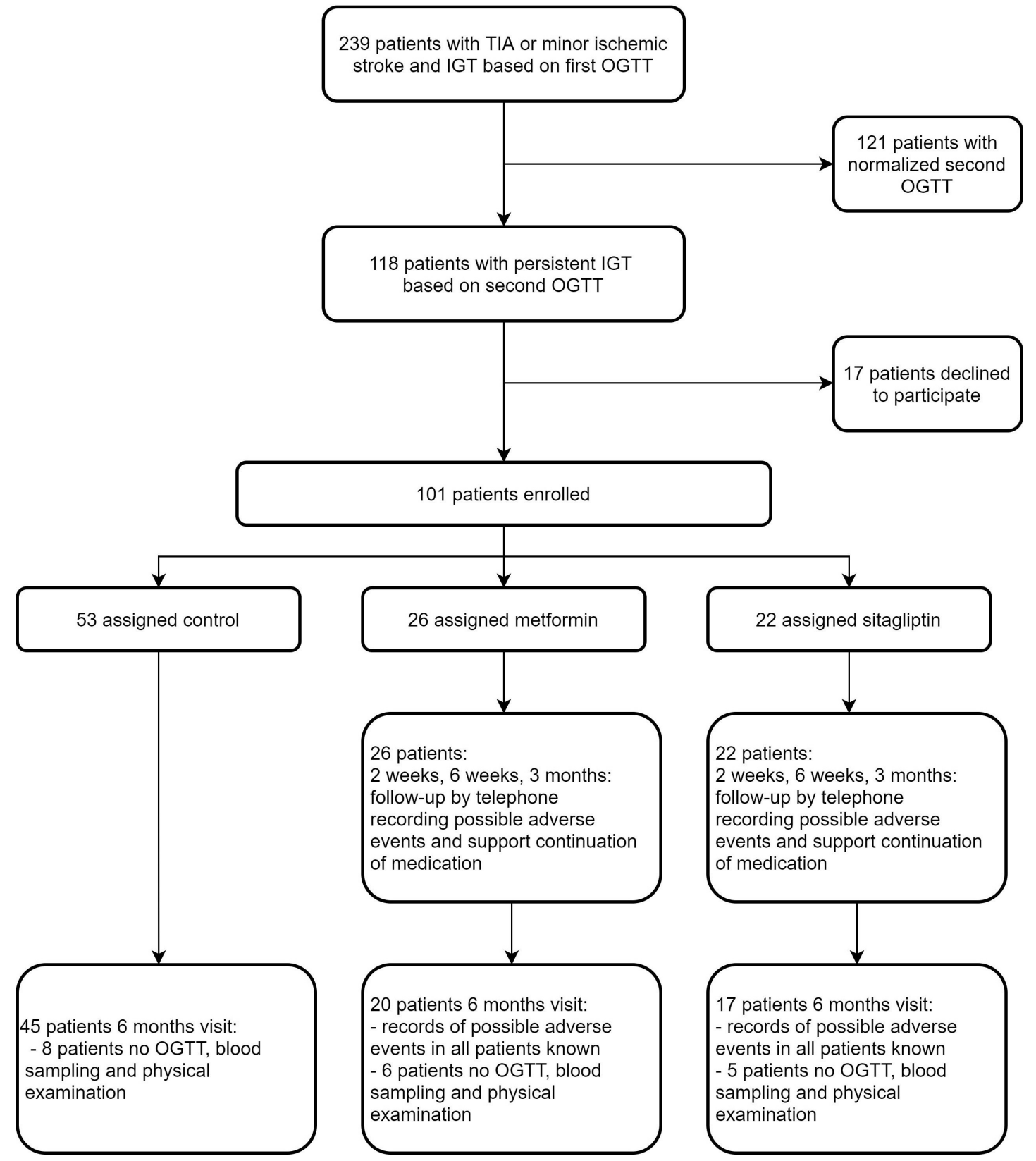

Figure 1 Flow chart of enrolment and follow-up. IGT, impaired glucose tolerance; OGTT, oral glucose tolerance test; TIA, transient ischaemic attack.

Of the total study population, 54 patients (53\%) were men, mean age was 68 years (SD 11), 55 patients $(54 \%)$ had ischaemic stroke, and $47(46 \%)$ had small vessel disease according to the TOAST criteria. The median time between the ischaemic event and randomisation was 51 days (IQR 38). Forty-seven patients (47\%) also had impaired fasting glucose levels and 38 patients $(37 \%)$ impaired HbAlc levels. The treatments groups were well matched, except for diastolic blood pressure and triglycerides levels, which were both lower in the metformin group (table 1 ).

Of 18 patients $(18 \%)$ the 6 months follow-up was not completed, and they did not undergo an OGTT, blood analysis, and physical examination; $8(15 \%)$ in the control group, $5(19 \%)$ in the metformin group and $5(23 \%)$ in the sitagliptin group ( $\mathrm{p}=0.72)$ (figure 1). Underlying reasons were lack of motivation to continue the study and logistic problems to visit the hospital again. The patients of whom the 6-month-follow up visit was not completed, were older ( 71 vs 67 years), more often female ( $56 \%$ vs $45 \%$ ), more often had ischaemic strokes instead of TIA ( $56 \%$ vs $43 \%$ ), and ischaemic cardiovascular diseases in their medical history ( $33 \%$ vs $24 \%$ ). These differences were not statistically significant (table 2 ).

At 6 months follow-up, patients with metformin had a mean 2-hour postload glucose level of $8 \mathrm{mmol} / \mathrm{L}$, with sitagliptin $8.1 \mathrm{mmol} / \mathrm{L}$ and with no medication $8.1 \mathrm{mmol} / \mathrm{L}$. The baseline adjusted difference in 2-hour postload glucose levels between treatment groups compared with control was not significant: $-0.04 \mathrm{mmol} / \mathrm{L}(95 \%$ CI -0.53 
Table 2 Baseline characteristics of patients with 6 months visit and patients with no 6 months visit

\begin{tabular}{|c|c|c|c|}
\hline & $\begin{array}{l}\text { Visit at } 6 \text { months, } \\
n=83\end{array}$ & $\begin{array}{l}\text { No visit at } 6 \\
\text { months, } n=18\end{array}$ & $P$ value \\
\hline Age in years, mean (SD) & $67(10)$ & $71(13)$ & 0.138 \\
\hline Men, n (\%) & $46(55)$ & $8(44)$ & 0.397 \\
\hline Days between event and OGTT, median (p25-p75) & $49(26-63)$ & 59 (27-82) & 0.313 \\
\hline \multicolumn{4}{|l|}{ Vascular risk factors } \\
\hline Current smoking, $\mathrm{n}(\%)$ & $16(19)$ & $1(6)$ & 0.158 \\
\hline $\mathrm{BMl}$ in $\mathrm{kg} / \mathrm{m}^{2}$, mean (SD) & $28(4)$ & 27 (3) & 0.195 \\
\hline Hypertension, n (\%) & $63(76)$ & $13(72)$ & 0.743 \\
\hline Hypercholesterolaemia, n (\%) & $73(88)$ & $16(89)$ & 0.911 \\
\hline Atrial fibrillation, $\mathrm{n}(\%)$ & $10(12)$ & $3(17)$ & 0.596 \\
\hline Systolic blood pressure in mm Hg, mean (SD) & $144(19)$ & $147(29)$ & 0.619 \\
\hline Diastolic blood pressure in mm Hg, mean (SD) & $81(11)$ & $75(16)$ & 0.079 \\
\hline \multicolumn{4}{|l|}{ Vascular history } \\
\hline Ischaemic cardiovascular disease, $\mathrm{n}(\%)$ & $20(24)$ & $6(33)$ & 0.416 \\
\hline \multicolumn{4}{|l|}{ Event } \\
\hline Ischaemic stroke, $\mathrm{n}(\%)$ & $36(43)$ & $10(56)$ & 0.347 \\
\hline TOAST classification & & & 0.584 \\
\hline Large artery atherosclerosis, $\mathrm{n}(\%)$ & $4(22)$ & $13(16)$ & \\
\hline Cardio embolism, n (\%) & $3(17)$ & $9(11)$ & \\
\hline Small vessel occlusion, $\mathrm{n}(\%)$ & $7(39)$ & $40(48)$ & \\
\hline Other determined aetiology, $\mathrm{n}(\%)$ & $1(6)$ & $1(1)$ & \\
\hline Unknown, n (\%) & $3(17)$ & $20(24)$ & \\
\hline \multicolumn{4}{|c|}{ Laboratory assessment during admission/visiting TIA outpatient clinic } \\
\hline Total cholesterol levels in mmol/L, mean (SD) & $4.8(1.1)$ & $4.8(1.2)$ & 0.850 \\
\hline LDL levels in mmol/L, mean (SD) & $3(0.9)$ & $2.9(0.9)$ & 0.851 \\
\hline HDL levels in mmol/L, mean (SD) & $1.5(0.7)$ & $1.3(0.4)$ & 0.224 \\
\hline Triglyceride levels in mmol/L, mean (SD) & $1.3(0.6)$ & $1.4(0.5)$ & 0.82 \\
\hline \multicolumn{4}{|l|}{ Glucose assessment during admission/visiting TIA clinic } \\
\hline Fasting glucose levels in mmol/L, mean (SD) & $5.6(0.7)$ & $5.4(0.8)$ & 0.209 \\
\hline 2 hour post-load glucose levels in mmol/L, mean (SD) & $8.9(0.9)$ & $8.9(1)$ & 0.793 \\
\hline HbA1c levels in mmol/L, mean (SD) & $38(2.7)$ & $37(3.3)$ & 0.134 \\
\hline Impaired fasting glucose $\geq 5.6 \mathrm{mmol} / \mathrm{L}, \mathrm{n}(\%)$ & $42(51)$ & $12(67)$ & 0.215 \\
\hline Impaired HbA1c levels $\geq 39$ mmol/L, n (\%) & $45(56)$ & $10(56)$ & 0.903 \\
\hline
\end{tabular}

BMI, body mass index; HbA1c, glycosylated Haemoglobin 1c; HDL, High-density lipoprotein; LDL, low-density lipoprotein; OGTT, oral glucose tolerance test; TIA, transient ischaemic attack; TOAST, Trial of Org 10172 in Acute Stroke Treatment.

to 0.45 ) (table 3 , figure 2). The difference with controls was not significant, either for metformin $(-0.13 \mathrm{mmol} / \mathrm{L}$; $95 \% \mathrm{CI}-1.14-0.87)$, or for sitagliptin $(-0.03 \mathrm{mmol} / \mathrm{L}$; $95 \% \mathrm{CI}-0.55-0.5)$. At 6 months, 17 patients $(39 \%)$ in the control group, 7 (33\%) in the metformin group and 7 $(41 \%)$ in the sitagliptin group (p 0.87) reverted to normal glucose tolerance (table 4).

Patients on metformin or sitagliptin had lower fasting glucose levels $(-0.21 \mathrm{mmol} / \mathrm{L} ; 95 \% \mathrm{CI}-0.36$ to -0.06$)$ and HbAlc levels $(-1.16 \mathrm{mmol} / \mathrm{mol} ; 95 \%$ CI -1.84 to -0.49$)$ than the control group (table 3, figure 2).
Overall, there was no significant reduction at 6 months in BMI, LDL levels and blood pressure compared with control (table 3).

Also, both sitagliptin and metformin showed a trend to a higher rate of conversion to normalised fasting glucose and $\mathrm{HbAlc}$ levels at 6 months compared with no medication (table 4).

\section{Prespecified subgroup analyses}

Patients who were on high dose metformin (1000 mg two times per day) did not have better outcomes regarding the 
Table 3 Mean baseline adjusted differences in primary and secondary outcome measures between treatment groups (metformin and sitagliptin combined compared with no medication)

\begin{tabular}{|c|c|c|}
\hline & Unadjusted & Adjusted \\
\hline $\begin{array}{l}\text { Baseline adjusted differences in } 2 \text { hour post-load glucose in } \mathrm{mmol} / \mathrm{L} \\
(95 \% \mathrm{Cl})\end{array}$ & $-0.06(-0.56$ to 0.44$)$ & $-0.04(-0.53$ to 0.45$)$ \\
\hline Baseline adjusted differences in fasting glucose levels in mmol/L $(95 \% \mathrm{Cl})$ & $-0.22(-0.37$ to -0.06$)$ & $-0.21(-0.36$ to -0.06$)$ \\
\hline $\begin{array}{l}\text { Baseline adjusted differences in } \mathrm{HbA} 1 \mathrm{c} \text { levels in } \mathrm{mmol} / \mathrm{mol} \\
(95 \% \mathrm{Cl})\end{array}$ & $-1.14(-1.8$ to -0.47$)$ & $-1.16(-1.84$ to -0.49$)$ \\
\hline Baseline adjusted differences in LDL levels in mmol/L (95\% Cl) & $-0.12(-0.28$ to 0.04$)$ & $-0.11(-0.27$ to 0.06$)$ \\
\hline Baseline adjusted differences in systolic blood pressure levels in $\mathrm{mm} \mathrm{Hg}(95 \% \mathrm{Cl})$ & $-2.91(-7.59$ to 1.77$)$ & $-2.46(-6.98$ to 2.06$)$ \\
\hline Baseline adjusted differences in diastolic blood pressure levels in $\mathrm{mm} \mathrm{Hg}(95 \% \mathrm{Cl})$ & $-1.8(-5.08$ to 1.47$)$ & $-1.57(-4.91$ to 1.78$)$ \\
\hline
\end{tabular}

Adjusted for age, sex, time to treatment, BMI and baseline glucose levels.

BMI, body mass index; HbA1c, glycosylated haemoglobin $1 \mathrm{c}$; LDL, low-density lipoprotein.

baseline adjusted differences than control patients. The same applied for the majority of patients with a combination of IGT, impaired fasting glucose and impaired $\mathrm{HbA1c}$ levels at randomisation. In addition, the on-treatment analyses mostly did not significantly differ from the intention to treat analyses (table 5).

\section{Adverse events}

The follow-up at 2 and 6 weeks, 3 months, and 6 months by telephone for recording of possible adverse events were completed in all patients on sitagliptin or metformin.

Thirteen patients $(50 \%)$ in the metformin group and $7(32 \%)$ in the sitagliptin group experienced side effects. The most common side effects were gastrointestinal complaints (table 6). There were no serious adverse events. Five patients ( $19 \%$ of the metformin group) lowered the metformin dose because of side effects.

A total of 24 patients discontinued the medication, of these $6(25 \%)$ experienced side effects. Of the 10 patients with medication who did not come for the 6 months visit, $3(30 \%)$ experienced side effects.

Sixteen patients $(61 \%)$ in the metformin group and 13 patients $(59 \%)$ in the sitagliptin group $(\mathrm{p}=0.86)$ were still on treatment after 6 months.

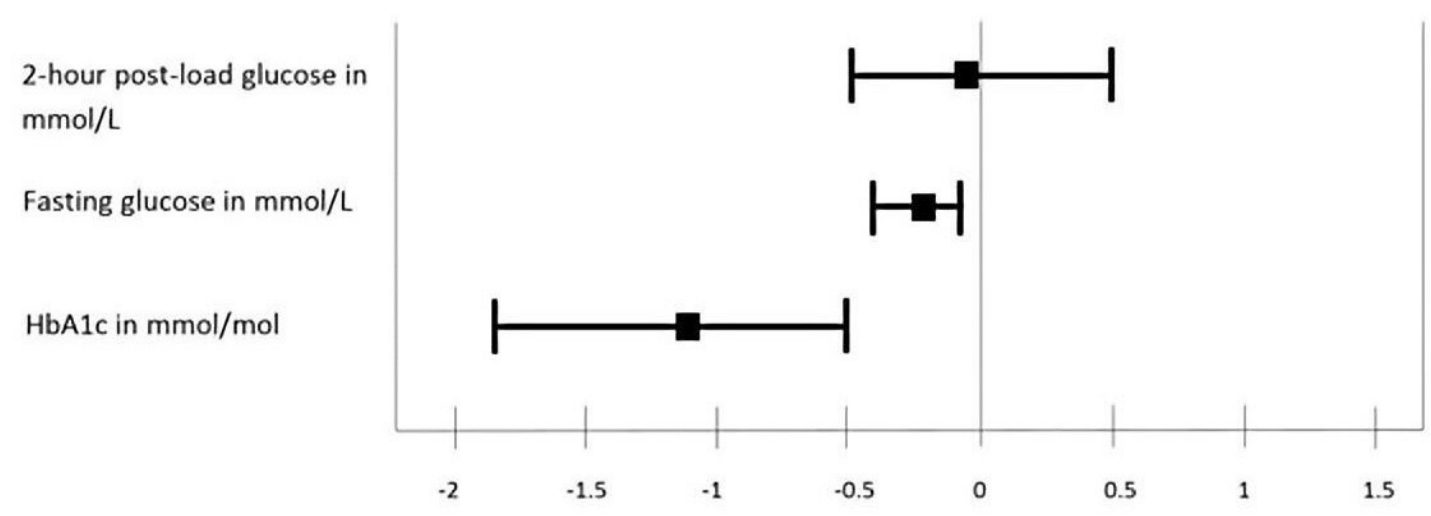

$\leftarrow$ Favours medication Favours control $\rightarrow$

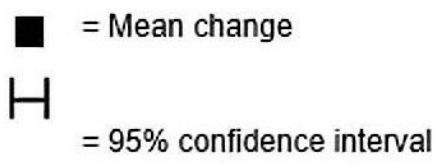

Adjustments for age, sex, time event to randomization, baseline BMI, baseline glucose levels

Figure 2 Mean change in glucose levels from baseline to 6 months with metformin or sitagliptin combined compared with no medication, $\mathrm{n}=87$. BMI, body mass index; HbA1c, glycosylated haemoglobin 1 $\mathrm{c}$. 


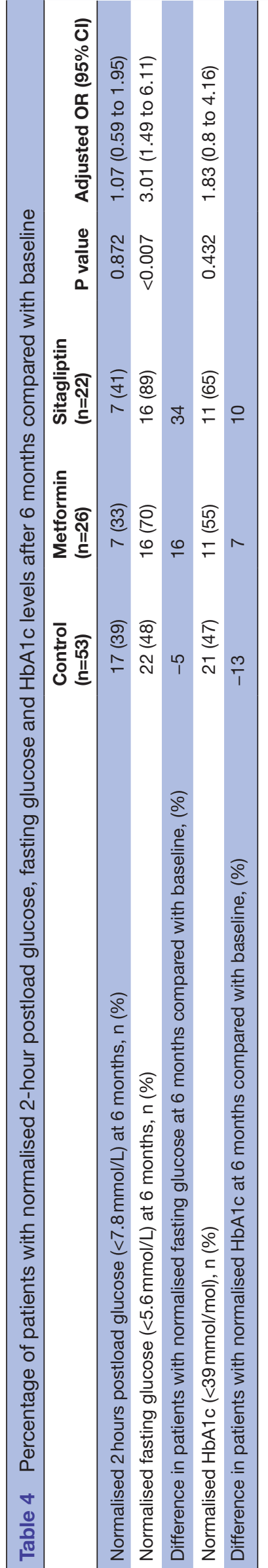

\section{DISCUSSION}

\section{Principal findings}

We found that metformin and sitagliptin had no significant effect on 2-hour postload glucose levels in patients with minor ischaemic stroke or TIA and IGT, but both moderately reduced fasting glucose and HbAlc levels. Although metformin and sitagliptin were both safe, metformin more often caused side effects, but not a higher rate of treatment discontinuation than sitagliptin.

\section{Interpretation in relation to other studies}

In contrast to our previous study, ${ }^{18}$ we did not find an effect of treatment on 2-hour postload glucose levels. We chose this outcome measure because 2-hour postload glucose levels detect more patients with impaired glucose metabolism than fasting glucose or HbAlc. ${ }^{27}$ However, an OGTT is not easy to reproduce accurately, due to interindividual and intraindividual variations, random variations of plasma glucose concentrations, and the effects of administration of hyperosmolar glucose concentration on gastric emptying. ${ }^{31}$

Interestingly, we did find a significant effect of metformin and sitagliptin on fasting glucose levels in a relative short period of 6 months and on HbAlc levels, which is a reflection of the average glucose levels of the past 2-3 months. In our previous study, we did not find a significant reduction in fasting glucose levels, maybe due to the shorter follow-up and smaller study population of our previous study. The HbA1c levels were not measured in our previous study.

Both studies reported a non-significant effect on BMI, lipid levels and blood pressure, while other studies report a decrease in weight, cholesterol levels and blood pressure with treatment with metformin or sitagliptin. ${ }^{19} 202432-34$ This might be explained by our relatively short follow-up period and small sample size.

We found a similar percentage of patients who experienced side effects, but a higher proportion of patients who discontinued metformin ( $40 \%$ in the current study vs $22 \%$ in our previous study). The slow increase in dosage of metformin and better information and support in our study did not lead to a decrease in occurrence of side effects, compared with our previous study. ${ }^{18}$

We compared our results to a study which assessed the efficacy and safety of sitagliptin compared with metformin in patients with type 2 diabetes. ${ }^{20}$ In our study, we found a higher incidence of side effects (32\% vs 6\%) and higher rates of discontinuation of sitagliptin ( $41 \%$ vs $12 \%)$. This might be explained by the fact that patients with TIA or ischaemic stroke are on average older, and the majority uses other drugs such as statins, antihypertensives and antithrombotic drugs, which may increase the risk of side effects.

Although statistically relevant, the reduction in glucose levels in our study was relatively small and the clinical relevance is not certain at this point. However, in our study, the study medication led to a higher rate of regression to normal glucose metabolism than no medication. Multiple studies have shown that regression to normal glucose tolerance is 
Table 5 Subgroup analyses of primary and secondary outcome measures between treatment groups

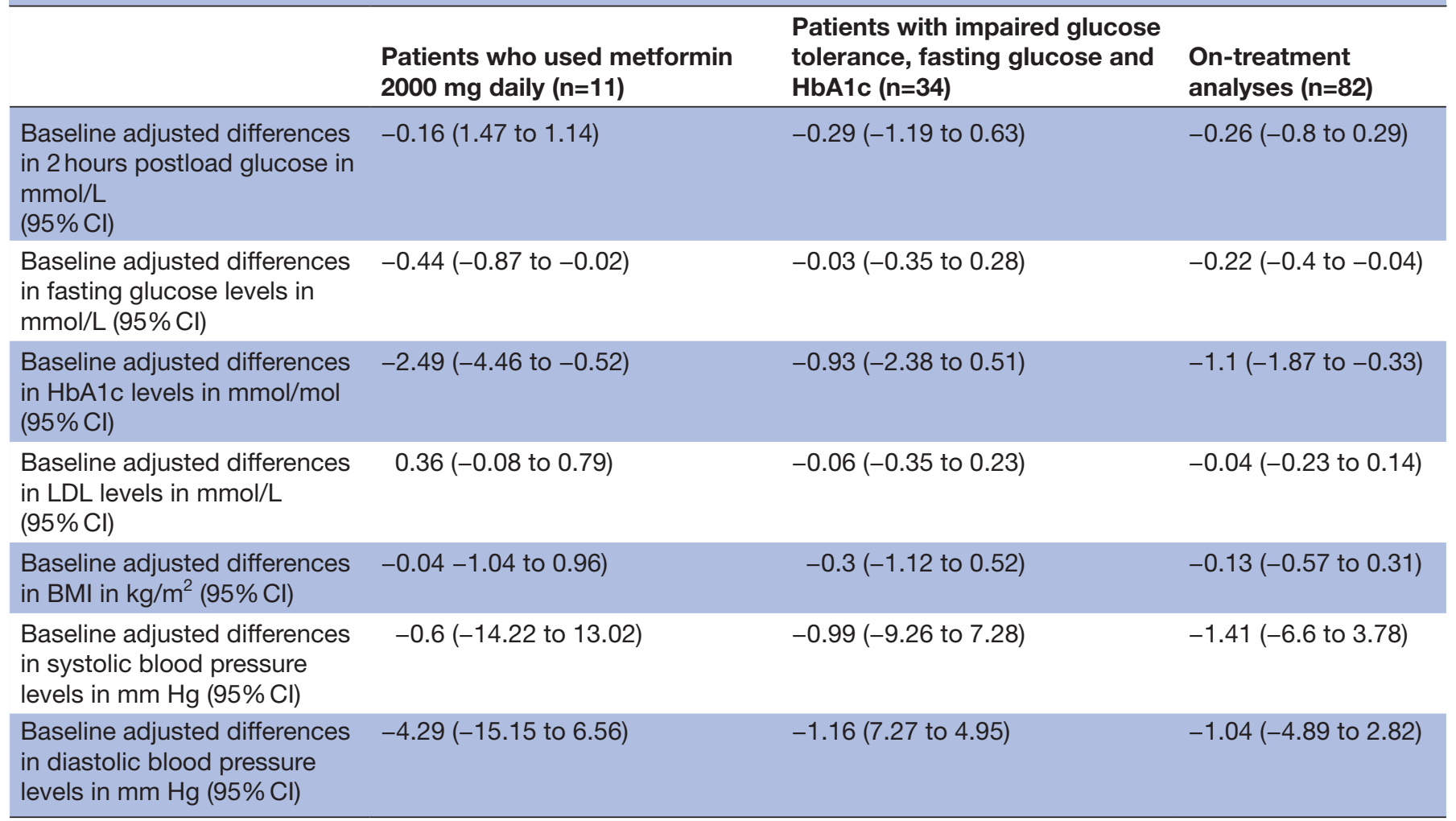

Adjusted for age, sex, time to treatment, BMI and baseline glucose levels.

$\mathrm{BMI}$, body mass index; HbA1c, glycosylated haemoglobin $1 \mathrm{c}$; LDL, low-density lipoprotein.

associated with reduction in cardiovascular risk during the time of intensive glucose lowering treatment, and that it possibly reduces mortality on the long term..$^{356}$

\begin{tabular}{|c|c|c|}
\hline & $\begin{array}{l}\text { Metformin, } \\
\mathrm{n}=26\end{array}$ & $\begin{array}{l}\text { Sitagliptin, } \\
\mathrm{n}=22\end{array}$ \\
\hline Patients with side effects, $n$ (\%) & $13(50)$ & $7(32)$ \\
\hline $\begin{array}{l}\text { Discontinuation of medication because of } \\
\text { side effects, } n(\%)\end{array}$ & $4(15)$ & $2(9)$ \\
\hline \multicolumn{3}{|l|}{ Type of side effects } \\
\hline Nausea, $\mathrm{n}$ & 8 & 2 \\
\hline Diarrhoea, $n$ & 8 & 0 \\
\hline Stomach ache, $\mathrm{n}$ & 6 & 2 \\
\hline Loss of appetite, $n$ & 4 & 1 \\
\hline Dry mouth, $\mathrm{n}$ & 1 & 2 \\
\hline Influenza like symptoms, $\mathrm{n}$ & 1 & 1 \\
\hline Muscle pain, $n$ & - & 2 \\
\hline Joint pain, $n$ & - & 1 \\
\hline Dizziness, $\mathrm{n}$ & - & 1 \\
\hline Vomiting, $n$ & 1 & - \\
\hline Headache, $\mathrm{n}$ & 1 & - \\
\hline Other, $\mathrm{n}$ & 7 & - \\
\hline
\end{tabular}

\section{Strengths and limitations}

Our study has limitations. First, our study had an open label design, and no placebo was used. However, outcome assessment was blinded for treatment allocation which decreased the risk of performance bias, and with this design, the study results are easier to relate to real life treatment strategies. We also did not study the effectiveness of the newer GLP1 analogues or SGLT2 inhibitors, which appeared to be more promising drugs to improve glucose metabolism as well as cardiovascular outcome.

Furthermore, a relatively large proportion of patients discontinued medication (40\%), despite support with frequent telephone calls. The majority of these patients did not report side effects as cause of the discontinuation. This finding is in concordance with results of other studies of preventive medication in this patient group. ${ }^{37}$ The patient compliance might even be less than reported, which can explain the relatively small effect on glucose levels we found.

Also, we had a relatively large proportion of patients who did not attend the 6 months visit. This could have impaired the power of our results and the clinical relevance. However, in all patients the frequency and nature of side effects was known.

\section{Clinical implications}

It is important to investigate the treatment options of IGT. IGT represents a disturbed metabolism, and interventions 
to improve this metabolism and reduce glucose levels might reduce the risk of cardiovascular disease in stroke patients with impaired glucose metabolism. Our study is one of the few which assessed whether glucose lowering therapy is effective in minor stroke patients with impaired glucose metabolism. A recent trial reported that pioglitazone can prevent cardiovascular disease in stroke patients with insulin resistance. ${ }^{16}$ A meta-analysis on glucoselowering pharmacological interventions in patients with IGT found possible beneficial effects on the incidence of stroke. ${ }^{17}$ Antidiabetic medication might not only have positive effects on glucose, but also on weight, cholesterol levels and blood pressure, and therefore, might be effective in the treatment of the other abnormalities of the metabolic syndrome. $19202432-34$

A recent study showed no long-term beneficial effect of intensive glucose lowering in patients with type 2 diabetes. ${ }^{36}$ So, although it is not known yet whether this will actually result in less (recurrent) cardiovascular morbidity and mortality, the results of our study could be important in optimising the secondary prevention in stroke patients with IGT, who have a high risk of recurrent stroke and other cardiovascular diseases. ${ }^{89}$

A phase III study is needed to investigate whether glucose lowering therapy is effective in reducing the incidence of recurrent stroke or other cardiovascular diseases. We found a significant effect of metformin and sitagliptin on fasting glucose levels and HbAlc levels, but not on 2-hour postload glucose levels. Fasting glucose levels and HbA1c levels are easy tests to perform. However, without the OGTT, more than half of patients with impaired glucose metabolism would be missed for inclusion.

Although there were more adverse events in the metformin group, the rate of discontinuation was the same in both treatment groups. Metformin is regarded as one of the most effective oral drugs in the management of type 2 diabetes. ${ }^{27} \mathrm{~A}$ recent randomised trial reported that sitagliptin did not have a significant effect on the incidence of cardiovascular diseases in patient with type 2 diabetes, thereby making sitagliptin a less suitable drug to investigate the long-term effects in pre-diabetes. ${ }^{34}$ Pioglitazone has also been proven effective as glucose lowering therapy in stroke patients, but pioglitazone gives a higher risk of weight gain, oedema and fracture. ${ }^{16}$

In the DPP-trial, the incidence of diabetes during 10 years follow-up was lower in the lifestyle group compared with the metformin group. This could mean that lifestyle modification is more effective than antidiabetic medication. ${ }^{38}$ The superior effect of lifestyle modification is possibly due to additional beneficial effects on the other components of the metabolic syndrome like disturbed cholesterol levels, high blood pressure and obesity. One can debate whether treatment should only focus on glucose lowering, or that treatment of all components of the metabolic syndrome are more effective.

Therefore, for a next phase III study, one might consider to compare the effect of lifestyle modification with metformin and/or one of the new promising drugs which actually reduce cardiovascular complications in patients with type 2 diabetes, like GLP1 analogues or SGLT2 inhibitors. ${ }^{21} 22$

In conclusion, metformin and sitagliptin are both effective and safe in reducing fasting glucose and HbAlc levels in patients with recent TIA or minor ischaemic stroke and IGT. However, the reduction of glucose levels and sample size was relatively small. The clinical relevance therefore needs to be tempered. The role of antidiabetic medication as treatment of IGT as a target for secondary prevention should be further explored.

Contributors EO, AZ, HL, DWJD and HdH analysed the data and drafted the manuscript. PJAMB, LJMMM and PK gave a critical review of the manuscript. All the authors have approved the final version of the manuscript.

Funding The authors have not declared a specific grant for this research from any funding agency in the public, commercial or not-for-profit sectors.

Competing interests None declared.

Patient and public involvement Patients and/or the public were not involved in the design, or conduct, or reporting, or dissemination plans of this research.

Patient consent for publication Not required.

Ethics approval The study protocol was approved by the central medical ethics committee and the research board of the participating centres, named Medisch Ethisch Toetsings Committee, number MEC-2012-085. All patients or their legal representatives gave written informed consent before randomisation. An independent data and safety monitoring board monitored the progress and safety of the trial, and performed an interim analysis. Based on this information, they advised the steering committee on prespecified criteria.

Provenance and peer review Not commissioned; externally peer reviewed.

Data availability statement Data are available on reasonable request. Individual participant data that underlie the results reported in this article after deidentification (text, tables, figures and appendices) will be shared. The study protocol, statistical analysis plan and analytical code will be available. The data will be available immediately and ending 5 years following article publication. The shared data are accessible for investigators whose proposed use of the data has been approved by an independent review committee identified for this purpose. The type of analyses is to achieve aims in the proposed proposal. Proposals should be directed to AZ, a.zandbergen@erasmusmc.nl. To gain access, data requestors will need to sign a data access agreement. A link for the shared data will be sent to the data requestors.

Open access This is an open access article distributed in accordance with the Creative Commons Attribution Non Commercial (CC BY-NC 4.0) license, which permits others to distribute, remix, adapt, build upon this work non-commercially, and license their derivative works on different terms, provided the original work is properly cited, appropriate credit is given, any changes made indicated, and the use is non-commercial. See: http://creativecommons.org/licenses/by-nc/4.0/.

ORCID iDs

Elizabeth Osei http://orcid.org/0000-0001-9405-0583

Diederik W J Dippel http://orcid.org/0000-0002-9234-3515

\section{REFERENCES}

1 Dave JA, Engel ME, Freercks R, et al. Abnormal glucose metabolism in non-diabetic patients presenting with an acute stroke: prospective study and systematic review. QJM 2010;103:495-503.

2 Matz K, Keresztes K, Tatschl C, et al. Disorders of glucose metabolism in acute stroke patients: an underrecognized problem. Diabetes Care 2006;29:792-7.

3 Kernan WN, Viscoli CM, Inzucchi SE, et al. Prevalence of abnormal glucose tolerance following a transient ischemic attack or ischemic stroke. Arch Intern Med 2005;165:227-33.

4 Fonville S, Zandbergen AAM, Koudstaal PJ, et al. Prediabetes in patients with stroke or transient ischemic attack: prevalence, risk and clinical management. Cerebrovasc Dis 2014;37:393-400.

5 Jia Q, Zheng H, Liu L, et al. Persistence and predictors of abnormal glucose metabolisms in patients after acute stroke. Neurol Res 2010;32:359-65. 
6 Vancheri F, Curcio M, Burgio A, et al. Impaired glucose metabolism in patients with acute stroke and no previous diagnosis of diabetes mellitus. QJM 2005;98:871-8.

7 Fonville S, den Hertog HM, Zandbergen AAM, et al. Occurrence and predictors of persistent impaired glucose tolerance after acute ischemic stroke or transient ischemic attack. J Stroke Cerebrovasc Dis 2014;23:1669-75.

8 Vermeer SE, Sandee W, Algra A, et al. Impaired glucose tolerance increases stroke risk in nondiabetic patients with transient ischemic attack or minor ischemic stroke. Stroke 2006;37:1413-7.

9 Lee M, Saver JL, Hong K-S. Effect of pre-diabetes on future risk of stroke: meta-analysis. BMJ 2012;344:e3564.

10 Oizumi T, Daimon M, Jimbu Y, et al. Impaired glucose tolerance is a risk factor for stroke in a Japanese sample--the Funagata study. Metabolism 2008;57:333-8.

11 Jia $Q$, Liu G, Zheng $\mathrm{H}$, et al. Impaired glucose regulation predicted 1-year mortality of Chinese patients with ischemic stroke: data from abnormal glucose regulation in patients with acute stroke across China. Stroke 2014;45:1498-500.

12 Steinberg $\mathrm{HO}$, Chaker $\mathrm{H}$, Leaming $\mathrm{R}$, et al. Obesity/insulin resistance is associated with endothelial dysfunction. Implications for the syndrome of insulin resistance. J Clin Invest 1996;97:2601-10.

13 Potter van Loon BJ, Kluft C, Radder JK, et al. The cardiovascular risk factor plasminogen activator inhibitor type 1 is related to insulin resistance. Metabolism 1993;42:945-9.

14 DeFronzo RA. Insulin resistance: a multifaceted syndrome responsible for NIDDM, obesity, hypertension, dyslipidaemia and atherosclerosis. Neth J Med 1997;50:191-7.

15 DeBoer MD, Gurka MJ, Woo JG, et al. Severity of the metabolic syndrome as a predictor of type 2 diabetes between childhood and adulthood: the Princeton lipid research cohort study. Diabetologia 2015;58:2745-52.

16 Kernan WN, Viscoli CM, Furie KL, et al. Pioglitazone after ischemic stroke or transient ischemic attack. N Engl J Med 2016;374:1321-31.

17 Hopper I, Billah B, Skiba M, et al. Prevention of diabetes and reduction in major cardiovascular events in studies of subjects with prediabetes: meta-analysis of randomised controlled clinical trials. Eur J Cardiovasc Prev Rehabil 2011;18:813-23.

18 den Hertog HM, Vermeer SE, Zandbergen AAM, et al. Safety and feasibiLIty of Metformin in patients with Impaired glucose Tolerance and a recent TIA or minor ischemic stroke (LIMIT) trial - a multicenter, randomized, open-label phase II trial. Int J Stroke 2015;10:105-9.

19 Arechavaleta R, Seck T, Chen Y, et al. Efficacy and safety of treatment with sitagliptin or glimepiride in patients with type 2 diabetes inadequately controlled on metformin monotherapy: a randomized, double-blind, non-inferiority trial. Diabetes Obes Metab 2011;13:160-8.

20 Aschner P, Katzeff HL, Guo H, et al. Efficacy and safety of monotherapy of sitagliptin compared with metformin in patients with type 2 diabetes. Diabetes Obes Metab 2010;12:252-61.

21 Kristensen SL, Rørth R, Jhund PS, et al. Cardiovascular, mortality, and kidney outcomes with GLP-1 receptor agonists in patients with type 2 diabetes: a systematic review and meta-analysis of cardiovascular outcome trials. Lancet Diabetes Endocrinol 2019;7:776-85.
22 Heerspink HJL, Perkins BA, Fitchett DH, et al. Sodium glucose cotransporter 2 inhibitors in the treatment of diabetes mellitus: cardiovascular and kidney effects, potential mechanisms, and clinical applications. Circulation 2016;134:752-72.

23 Gillies CL, Abrams KR, Lambert PC, et al. Pharmacological and lifestyle interventions to prevent or delay type 2 diabetes in people with impaired glucose tolerance: systematic review and metaanalysis. BMJ 2007;334:299-302.

24 Knowler WC, Barrett-Connor E, Fowler SE. Reduction in the incidence of type 2 diabetes with lifestyle intervention or metformin. N Engl J Med 2006;346:393-403.

25 Osei E, Fonville S, Zandbergen AAM, et al. Metformin and sitAgliptin in patients with impAired glucose tolerance and a recent TIA or mino ischemic stroke (MAAS): study protocol for a randomized controlled trial. Trials 2015;16:1-6.

26 van Swieten JC, Koudstaal PJ, Visser MC, et al. Interobserver agreement for the assessment of handicap in stroke patients. Stroke 1988;19:604-7.

27 American Diabetes Association. Standards of medical care in Diabetes-2018. Diabetes Care 2018;41:S55-64.

28 Alberti K, Zimmet P. Definition, diagnosis and classification of diabetes mellitus and its complications: report of a who consultation. Geneva. Diabet Med 1998;15:539-53.

29 Powers WJ, Rabinstein AA, Ackerson T, et al. 2018 guidelines for the early management of patients with acute ischemic stroke: a guideline for healthcare professionals from the American heart Association/ American stroke association. Stroke 2018;49:46-110.

30 Vickers AJ. The use of percentage change from baseline as an outcome in a controlled trial is statistically inefficient: a simulation study. BMC Med Res Methodol 2001;1:1-4.

31 Ko GT, Chan JC, Woo J, et al. The reproducibility and usefulness of the oral glucose tolerance test in screening for diabetes and other cardiovascular risk factors. Ann Clin Biochem 1998;35 (Pt 1:62-7.

32 Lundby-Christensen L, Tarnow L, Boesgaard TW. Metformin versus placebo in combination with insulin analogues in patients with type 2 diabetes mellitus - the randomised, blinded Copenhagen insulin and metformin therapy (CIMT) trial. BMJ Open 2015;6:1-11.

33 Derosa G, Tritto I, Romano D, et al. Effects of sitagliptin on lipid profile in patients with type 2 diabetes mellitus after 7 years of therapy. J Clin Pharmacol 2019;59:1391-9.

34 Green JB, Bethel MA, Armstrong PW, et al. Effect of sitagliptin on cardiovascular outcomes in type 2 diabetes. $N$ Engl J Med 2015;373:232-42.

35 Perreault L, Temprosa M, Mather KJ, et al. Regression from prediabetes to normal glucose regulation is associated with reduction in cardiovascular risk: results from the diabetes prevention program outcomes study. Diabetes Care 2014;37:2622-31.

36 Reaven PD, Emanuele NV, Wiitala WL, et al. Intensive glucose contro in patients with type 2 diabetes - 15-year follow-up. $N$ Engl J Med 2019;380:2215-24.

37 Cheiloudaki E, Alexopoulos E. Adherence to treatment in stroke patients. Int J Environ Res Public Health 2019;16:196-11.

38 Knowler WC, Fowler SE, et al, Diabetes Prevention Program Research Group. 10-Year follow-up of diabetes incidence and weight loss in the diabetes prevention program outcomes study. Lancet 2009;374:1677-86. 